\title{
Purification of transcriptionally active multimeric plasmid DNA using zwitterionic detergent and carbonate apatite nano-particles.
}

\begin{abstract}
Plasmid DNA is one of the indispensable components in molecular biology research and a potential biomaterial for gene therapy and DNA vaccination. Both quality and quantity of extracted plasmid DNA are of the great interests in cloning and subsequent expression of genes in vitro and in vivo for basic research and therapeutic interventions. Bacteria with extremely short generation times are the valuable source of plasmid DNA that can be isolated through a number of existing techniques. However, the current methods have some limitations in isolating high quality plasmid DNA since the multimeric plasmid which is believed to be more efficiently transcribed by RNA polymerase than the monomeric form, is almost lost during the extraction process. Recently, we developed a rapid isolation technique for multimeric plasmid based on generation of a 'protein aggregate' using a zwitterionic detergent and alkali. Here we have investigated the roles of different parameters in the whole extraction process to optimise the production of high quality multimeric plasmid DNA. Moreover, we have showed the advantageous effects of nanoparticles to effectively sediment the 'protein aggregate' for smooth elution of multimeric plasmid DNA from it. Finally, quality assessment study has revealed that the isolated multimeric DNA is at least 10 times more transcriptionally active than the monomeric form isolated by the commercially available Qiaget kit.
\end{abstract}

Keyword: Carbonate apatite; Nano-crystals; Particle size; NaCl; Glucose; Transfection. 